Article

\title{
Metaheuristic optimization methods for optimal power flow analysis in DC distribution networks*
}

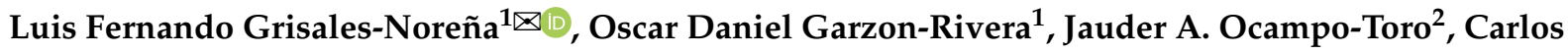 \\ Andrés Ramos-Paja ${ }^{3}{ }^{\circledR}$, Miguel Angel Rodriguez Cabal ${ }^{2}$ \\ 1 Instituto Tecnológico Metropolitano, Laboratorio de Energía Eléctrica y Potencia, Medellín, Colombia. \\ 2 Instituto Tecnológico Metropolitano, Maestría en Gestion Energética Industrial, Medellín, Colombia. \\ 3 Universidad Nacional de Colombia, Facultad de Minas, Medellín, Colombia. \\ 凶luisgrisales@itm.edu.co.
}

Received: 13 February 2020; Accepted: 27 March 2020; Published: 17 December 2020

\begin{abstract}
In this paper is addressed the optimal power flow problem in direct current grids, by using solution methods based on metaheuristics techniques and numerical methods. For which was proposed a continuous nonlinear programming problem, that describes the optimal power flow problem in direct current grids. As solution methodology was proposed a master-slave strategy, which used in master stage three continuous solution methods for solving the optimal power flow problem: a particle swarm optimization algorithm, a continuous version of the genetic algorithm and the black hole optimization method. In the slave stages was used a numerical method based on successive approximations for solving the power flow problem, this method is used to calculates the objective function associated with each solution proposed by the master stage. As objective function was used the reduction of power loss on the electrical grid, associated to the energy transport. To validate the solution methodologies proposed were used two test systems of 21 and 69 buses, respectively; by implementing three levels of maximum distributed power penetration: $20 \%, 40 \%$ and $60 \%$ of the power supplied by the slack bus, without considering distributed generators installed on the electrical grid. All simulations were carried out in software Matlab, results obtained by demonstrating that the methods with the best performance was the $\mathrm{BH} / \mathrm{SA}$, due to that show the best trade-off between the reduction of the power loss and processing time, for solving the optimal power flow problem in direct current networks.
\end{abstract}

Keywords: Optimization algorithms, direct current networks, optimal power flow, particle swarm optimization, black-hole optimization, genetic algorithms.

Resumen: En este artículo se aborda el problema de flujo óptimo de potencia (OPF, de sus siglas en inglés) en redes de corriente continua, empleando métodos de solución basados en técnicas Metaherísticas y métodos numéricos. Para lo cual se plantea una formulación matemática no lineal continua, la cual describe el problema de flujo óptimo de potencia en redes de corriente continua. Como alternativa de solución se propone una metodología hibrída basada en una estrategia maestro-esclavo, la cual emplea dentro de la etapa maestra tres métodos de solución para resolver el problema de OPF: un algoritmo de

\footnotetext{
${ }^{*}$ How to cite this article: Grisales Noreña, L., Garzón Rivera, O., Ocampo Toro, J., Ramos Paja, C., Rodriguez Cabal, M. (2020). Metaheuristic Optimization Methods for Optimal Power Flow Analysis in DC Distribution Networks. Transactions on Energy Systems and Engineering Applications, 1(1), 13-31. DOI: 10.32397/tesea.vol1.n1.2 ISSN: 2745-0120
} 
optimización por cúmulo de partículas, un algoritmo genético continuo y un algoritmo de optimización basado en agujeros negros. Dentro de la etapa esclava es empleado un método basado en aproximaciones sucesivas para resolver el problema de flujo de carga, encargado de calcular la función objetivo de cada posible solución propuesta por la etapa maestra. Como fución objetivo se emplea la reducción en las pérdidas de potencia en el sistema eléctrico, asociadas al transporte de energía. Para la validación de los métodos propuestos, se emplearon los sistemas de prueba de 21 y de 69 nodos, los cuales fueron evaluados bajo niveles de penetración de potencia de $20 \%, 40 \%$ y $60 \%$ de la potencia total generada por el nodo Slack, bajo condiciones nulas de generación distribuida. Las simulaciones ejecutadas vía Matlab, demostraron que el método con el mejor desempeño es el BH/SA, ya que presenta el mejor balance entre reducción en pérdidas de potencia y tiempo de procesamiento requerido para resolver el problema de OPF en redes de corriente continua.

Palabras claves: Algoritmos de optimización, redes de corriente continua, flujo de potencia óptimo, optimización por cúmulo de partículas, optimización basada en agujeros negros, algoritmos genéticos.

\section{Introducción}

Dada la importancia de las redes de CC en los últimos años y la necesidad de integrar recursos renovables en los sistemas de producción de energía eléctrica, reducir el impacto negativo de los combustibles fósiles y mejorar diferentes aspectos técnico-económicos (Garces 2017), multiples investigadores han evaluado y estudiado el efecto de la integración de generadores distribuidos (GDs) en estos tipos de red. Para analizar el impacto de la energía suministrada por los GDs en la red de CC, en la literatura se han propuesto diferentes métodos que permiten determinar niveles óptimos de inyección de energía por cada generador en la red, con el fin de mejorar los diferentes indicadores técnicos-economicos que definen la red eléctrica, tales como: pérdidas de potencia, perfiles de voltaje, niveles de emisión de $\mathrm{CO}_{2}$, entre otros (Gil-González et al. 2019). Un ejemplo de esto se puede apreciar en (Li et al. 2018; Montoya et al. 2019), en donde se propone una formulación de programación conica de segundo orden y un modelo cuadrático convexo para resolver el problema de OPF en redes de CC independientes, respectivamente. En ambos casos se propone la reducción de la pérdidas de potencia como función objetivo. Por otro lado, en (Nasir et al. 2018) se utilizó la función Linprog para dar solución al problema de OPF en redes de CC con topología radial. El principal problema de los métodos citados anteriormente es el uso de un software de optimización especializado para resolver el problema CC OPF, lo cual representa un problema debido a lo costoso que resulta acceder a este tipo de software.

En (Wang et al. 2018), los autores con el objetivo de evitar el uso de software especializado para resolver el problema de OPF en redes de CC, propusieron una metodología basada en el algoritmo de optimización por cúmulo de partículas (PSO, por sus siglas en inglés). Sin embargo, este trabajo presenta un problema, puesto que los autores consideran una microrred (MR) con un nodo único que desprecia las múltiples ramas y nodos existentes en las MR CC regulares, i.e., las que se encuentran regularmente en la industria y literatura (Grisales-Noreña et al. 2020). En (Velasquez et al. 2019) se aplicó el algoritmo de optimización basado en agujeros negros $(\mathrm{BH}$, de sus siglas en ingés) para resolver el problema de $\mathrm{OPF}$, empleando como función objetivo la reducción de pérdidas de potencia. En este trabajo se compara la efectividad de la metodología de solución propuesta con otros metodos heurísticos, sin realizar comparaciones en cuanto a los tiempos de procesamiento requeridos por estas. Adicionalemente, en (Montoya et al. 2018a), se presenta la aplicacion de un algoritmo genetico continuo (CGA, por sus siglas en inglés) para resolver el problema de flujo de potencia óptimo en redes CC. Empleando el software 
GAMS/CONOPT para demostrar la efectividad de la metodología propuesta. En este trabajo los autores no analizan los tiempos de procesamiento.

Dentro de la revisión del estado del arte realizada en este documento, se determinó que fueron pocos los trabajos hallados que aborden el problema de OPF en redes de CC sin usar software especializado, lo que demuestra la necesidad de proponer técnicas basadas en programación secuencial que permitan obtener soluciones con alta calidad y con tiempos de procesamiento cortos. En base a esa situación, en este artículo se propone una metodología maestro-esclavo basada en programación secuencial. Para la etapa maestra, se prueban tres métodos diferentes de optimización (BH, CGA y PSO), que se encargan de dimensionar los GDs ubicados dentro de la red de CC (Grisales-Noreña et al. 2019). Para la etapa esclava, se utilizó el método de SA, descrito y analizado profundamente en (Montoya et al. 2019). Siendo la tarea de la etapa esclava resolver el problema de flujo de carga, aplicando las posibles soluciones propuestas en la etapa maestra, y de esta manera determinar la configuración óptima de los GDs que permita la mayor reducción en pérdidas de potencia de la red de CC. Al combinar los métodos de optimización con el SA, se obtienen tres metodologías maestro-esclavo diferentes: BH/SA, CGA/SA y PSO/SA.

Para validar la efectividad y robustez de las diferentes metodologías de solución propuestas en este documento, se utilizaron los sistemas de prueba de 21 y 69 nodos. Además, se consideraron tres escenarios diferentes de penetración de potencia para los GDs como la energía máxima capaz de suministrar cada GD a la red. Esos valores equivalen al 20\%, 40\% y 60\%, de la energía total generada por el nodo Slack. Estos escenario de inyección de potencia, permiten evaluar el impacto de la generación de energía distribuida en el sistema eléctrico. Todas las simulaciones se hicieron en el software MATLAB, con el objetivo de proporcionar las mismas condiciones de prueba para todos los métodos de solución empleados, y de esta manera determinar el método con el mejor desempeño en términos de función objetivo y tiempo de procesamiento.

Este documento se encuentra organizado de la siguiente forma: la sección 2 presenta la formulación matemática del problema de flujo óptimo de potencia en redes de CC. La sección 3 define la metodología propuesta basada en la estrategia maestro-esclavo. La sección 4 presenta las características principales de los sistemas de prueba y muestra los parámetros de validación numérica de los tres algoritmos de optimización propuestos en este artículo, con su respectivo análisis y discusión. Finalmente, la sección 5 presenta los comentarios finales y trabajo futuro derivados de este trabajo.

\section{Formulación Matemática}

El problema de OPF se formula a través de un modelo matemático compuesto por una función objetivo relacionada con un conjunto de restricciones del flujo de energía en redes de CC (Montoya et al. 2018b). Este modelo matemático puede usarse para satisfacer diferentes objetivos técnicos, económicos, operativos y ambientales fijados por el propietario u operador de la red eléctrica; por ejemplo, las pérdidas de potencia asociadas al transporte de enérgia, que es el indicador técnico seleccionado como función objetivo en este artículo.

\subsection{Función objetivo}

La función objetivo se define como un valor a modificar, supervisar o en esta caso especf́ico, a minimizar. En este artículo fue seleccionada como función objetivo la minimización de las pérdidas de potencia dentro de la MR de CC, las cuales se encuentran asociadas al transporte de energía por las ramas; esto se logra empleando la herramienta de flujo óptimo de potencia-OPF. Para representar dicha función, se utiliza la ecuación (1), donde $P_{\text {loss }}$ representa las pérdidas de potencia en la red (la variable que se debe 
minimizar), la cual está en función de $v$ y $G_{B}$, las cuales representan los voltajes nodales y la matriz de conductancia asociada a las ramas, respectivamente.

$$
\min P_{\text {loss }}=\min \left(v^{T} G_{B} v\right),
$$

\subsection{Restricciones}

Las restricciones se refieren a los límites y parámetros que se deben respetar para los problemas de flujo de potencia y OPF, y se representan a través de las siguientes ecuaciones:

$$
\begin{array}{r}
p_{g}+p_{g d}-p_{L}=\mathbf{D}(v) \mathbf{G} v \\
P_{g}^{\text {min }} \leq p_{g} \leq P_{g}^{\max } \\
P_{g d}^{\text {min }} \leq p_{d g} \leq P_{g d}^{\text {max }} \\
V^{\text {min }} \leq v \leq V^{\text {max }} \\
\text { Ones }^{T} p_{g d}-P_{G D}^{\max } \leq 0
\end{array}
$$

La interpretación matemática de las ecuaciones (2) a (6) se hace de la siguiente manera: en la ecuación (2) $p_{g}, p_{g d} \mathrm{y} p_{L}$ se refieren a la potencia generada por el nodo Slack, la potencia suministrada a la red por cada GD y la potencia demandada en los nodos de la red, respectivamente. Esta ecuación expresa el balance de potencia en la red eléctrica. En las ecuaciones (3) y (4), $P_{g}^{\min }$ y $P_{g}^{\max }$ denotan la potencia mínima y máxima que el nodo Slack puede entregar a la red. Del mismo modo, $P_{g d}^{\min }$ y $P_{g d}^{\max }$ definen la potencia mínima y máxima que los GDs pueden inyectar a la red. Estas ecuaciones se utilizan para determinar la capacidad de generación tanto del nodo Slack como de los GDs. La ecuación (5) incluye $V^{\text {min }}$ y $V^{\text {max }}$, estos términos matemáticos representan los límites de regulación de voltaje. Finalmente, la ecuación (6) define la generación máxima permitida a los GDs en la red de CC, donde $P_{G D}^{\max }$ representa la generación distribuida permitida con respecto a la potencia generada por el nodo Slack, sin considerar la ubicación del generador distribuido en el sistema eléctrico. En esta ecuación ones ${ }^{T}$ es un vector que contiene únicamente unos transpuestos, esto permite realizar la operación matemática entre $p_{g d}$ y $P_{G D}^{\max }$.

\subsection{Función adaptación:}

La función adaptación (z) se compone del valor de la función objetivo original, así como de las restricciones agregadas mediante penalizaciones. El valor de la función adaptación propuesta en este documento para guiar el problema de optimización en la etapa maestra, se expresa de la siguiente manera:

$$
\min z=\left(\begin{array}{l}
p_{\text {loss }}+\beta_{1} \text { Ones }^{T} \max \left\{0, v-V^{\max }\right\} \\
-\beta_{2} \text { Ones }^{T} \min \left\{0, v-V^{\min }\right\} \\
+\beta_{3} \text { Ones }^{T} \max \left\{0, p_{g d}-P_{g d}^{\max }\right\} \\
-\beta_{4} \text { Ones }^{T} \min \left\{0, p_{g d}-P_{g d}^{\min }\right\} \\
\left.+\beta_{5} \max \left\{0, \text { Ones }^{T} p_{g d}-P_{G D}^{\max }\right)\right\}
\end{array}\right)
$$

donde $\beta_{1}$ a $\beta_{5}$ corresponden a factores de penalización, que suelen ser superiores a cero. En este documento, cada factor de penalización es igual a 1000 para obligar a los métodos de optimización a 
cumplir todas las condiciones impuestas sobre el problema de OPF formulado desde (1) a (6). Dicho valor se obtuvo mediante prueba y error, cuando se cumplen todas las restricciones, todos los factores de penalización deben ser anulados por funciones $\max \{\cdot\}$ y $\min \{\cdot\}$, lo que convierte el valor de la función adaptación en una función objetivo, ya que en ese caso $z$ es igual a $P_{\text {loss }}$.

\section{Metodología Propuesta}

El conjunto de ecuaciones presentada en la seccioń 2 requiere métodos no lineales para determinar su solución. En ese sentido, se propone dividir el problema del flujo de potencia óptimo en dos etapas: la primera etapa (maestra) utiliza tres métodos diferentes de optimización para determinar el nivel de inyección de potencia óptima de los DGs. La segunda etapa (esclava) usa el SA para evaluar la función de aptitud en cada una de las posibles soluciones propuestas por la etapa maestra. Las siguientes subsecciones describen la metodología maestro-esclavo empleada en este documento:

\subsection{Etapa maestra: algoritmos de optimización}

La etapa maestra se encarga del dimensionamiento de los DGs. Este proceso se realiza con tres técnicas diferentes de optimización: BH, CGA y PSO. Estos métodos fueron seleccionados debido a los excelentes resultados reportados en la literatura especializada, cuando fueron empleados para solucionar el problema de OPF en redes de CA y CC (Hasan and El-Hawary 2014; Montoya et al. 2018a; Moradi and Abedini 2012; Velasquez et al. 2019; Wang et al. 2018). Por otro lado, con el objetivo de reducir el tiempo de procesamiento y proporcionar una comparación equitativa entre los métodos utilizados, se utilizó el método de SA para resolver los flujos de potencia requeridos para determinar la viabilidad de la solución propuesta por cada método de optimización. Los métodos de solución empleados en la etapa esclava se presentan a continuación.

\subsubsection{Método de optimización de agujeros negros $(\mathrm{BH})$ :}

Esta es una técnica de optimización inspirada en la naturaleza y basada en la interacción dinámica entre estrellas y agujeros negros (Bouchekara 2014). Esta técnica se ha utilizado para resolver problemas de optimización no lineales mediante la implementación de un enjambre de partículas (estrellas), así como un criterio de eliminación y generación de estrellas a través de un enfoque heurístico (radio del horizonte de eventos). El método BH se presenta brevemente a continuación resaltando los pasos más importantes para su implementación computacional.

\section{Nacimiento de las estrellas:}

El BH es un algoritmo de optimización basado en la población, derivado del optimizador convencional basado en enjambre de partículas (Piotrowski et al. 2014); en ese sentido, la población inicial $\left(P_{t}\right)$ corresponde al primer conjunto de estrellas distribuidas aleatoriamente sobre el espacio de solución, es decir, como un cúmulo de estrellas en el universo (Bouchekara 2013). Durante la generación de este conjunto de estrellas, el número de estrellas $\left(\left(n_{i}\right)\right.$ - posibles soluciones), es el número de filas; mientras que el número de GD $\left(n_{g d}\right)$ es el número de columnas, tal como se formula en la Ecuación (8).

$$
P_{t}=P_{g d}^{\min } o\left(n_{i}, n_{g d}\right)+\left(P_{g d}^{\max }-P_{g d}^{\min }\right) r\left(n_{i}, n_{g d}\right)
$$

donde $P_{t}$ representa la matriz de población estelar, $o\left(n_{i}, n_{g d}\right)$ es una matriz rectangular llena de unos, y $r\left(n_{i}, n_{g d}\right)$ corresponde a una matriz rectangular llena de números aleatorios de cero a uno con 
propiedades de distribución normales. Se debe tener en cuenta que $P_{t}$ corresponde a la población actual y cada individuo (estrella) en su interior representa la generación de energía total en todos los nodos que contienen GDs. Además, la mejor solución (función de condición física más baja para problemas de minimización) dentro de la población inicial $P_{t}$ se selecciona como la ubicación del agujero negro. En el caso del problema del flujo de potencia de redes de CC, $P_{t}$ tiene la siguiente estructura:

$$
P_{t}=\left[\begin{array}{cccccc}
p_{g d}(1,1) & p_{g d_{(1,2)}} & \cdots & p_{g d}(1, k) & \cdots & p_{g d}\left(1, n_{g d}\right) \\
p_{g d}(2,1) & p_{g d}(2,2) & \cdots & p_{g d}(2, k) & \cdots & p_{g d}\left(2, n_{g d}\right) \\
\vdots & \vdots & \ddots & \vdots & \vdots & \vdots \\
p_{g d}(l, 1) & p_{g d_{(1,2)}} & \cdots & p_{g d}(l, k) & \cdots & p_{g d}\left(l, n_{g d}\right) \\
\vdots & \vdots & \vdots & \vdots & \ddots & \vdots \\
p_{g d}\left(n_{i}, 1\right) & p_{g d_{\left(n_{i}, 2\right)}} & \cdots & p_{g d}\left(n_{i}, k\right) & \cdots & p_{g d}\left(n_{i}, n_{g d}\right)
\end{array}\right]_{n_{i} \times n_{g d}}
$$

donde $p_{g d(l, k)}$ representa la potencia activa generada por el generador $k$ en la solución individual $l$.

\section{Movimiento de estrellas:}

El comportamiento dinámico de las estrellas en la proximidad de un agujero negro está muy influenciado por la intensa fuerza gravitacional de este último. En ese sentido, el movimiento de cualquier estrella puede tener un comportamiento particular en función de su ubicación con respecto a la posición del agujero negro (Bouchekara 2014). Tal comportamiento es emulado por la relación matemática presentada en la Ecuación (9).

$$
P_{t+1}^{i}=p_{t}^{i}+\left(p_{t}^{B H}-p_{t}^{i}\right) r\left(1, n_{g d}\right) ; \quad i=1,2, \ldots, n_{i}
$$

donde $p_{t}^{B H}$ representa el agujero negro en la población $t, \mathrm{y} P_{t+1}^{i}$ denota el individuo $i^{\text {th }}$ después de su movimiento. Después de este proceso, la ubicación del agujero negro permanece inalterada.

\section{Actualización del agujero negro:}

Después de generar la población descendente de estrellas $P_{t+1}$, la ubicación del agujero negro debe cambiarse si el individuo $i^{\text {th }}$ entre la población descendente exhibe un valor de función daptación menor que el agujero negro actual, es decir, $p_{t}^{B H}=p_{t+1}^{i}$; de lo contrario, la ubicación del agujero negro permanece constante, $p_{t+1}^{B H}=p_{t}^{B H}$.

\section{Reemplazo de estrella:}

La supervivencia de una estrella en el vecindario de un agujero negro depende de su posición actual con respecto a la ubicación del agujero negro. En física teórica, cualquier objeto que cruza el horizonte de eventos alrededor de un agujero negro está destinado a la destrucción. Sin embargo, este escenario catastrófico genera material estelar que permite la formación de nuevas estrellas. Para emular la posibilidad de que una estrella arbitraria en la población descendente sea absorbida por el agujero negro, el radio del horizonte de sucesos se define como: 


$$
R_{E H}=\frac{f\left(P_{t+1}^{B H}\right)}{\sum_{i=1}^{n_{i}} f\left(P_{t+1}^{i}\right)},
$$

donde $f\left(P_{t+1}^{B H}\right)$ representa el mejor valor de la función adaptación de todos los individuos contenidos en la población actual, mientras que el denominador de (10) corresponde a la suma de la función de aptitud de todos los individuos en la misma iteración.

Para determinar si alguna estrella cruza el horizonte de sucesos, la distancia euclidiana de dicha estrella con respecto a la ubicación del agujero negro se define en la ecuación (11).

$$
D_{B H-i}=\left\|P_{t+1}^{B H}-P_{t+1}^{i}\right\|
$$

Si $R_{E H}>D_{B H-i}$, se genera aleatoriamente una nueva estrella para reemplazar la absorbida por el agujero negro; de lo contrario, la estrella continúa en la población actual. Observe que el nacimiento de nuevas estrellas aumenta la posibilidad de expandir la exploración del algoritmo sobre el espacio de la solución, lo que se consideraría exploración global (Bouchekara 2013).

\section{Criterio de parada:}

Para detener la exploración del BH sobre el espacio de solución, se debe cumplir una de las siguientes condiciones:

- El número máximo de iteraciones $\left(t_{\max }\right)$ ha sido alcanzado.

- Después de $k$ iteraciones consecutivas, el agujero negro no actualiza su posición.

Finalmente, el Algoritmo 1 muestra la aplicación del método de solución maestro-esclavo propuesto en este estudio para resolver problemas de OPF en redes de CC a través de un enfoque híbrido BH-SA.

\subsubsection{Algoritmo genético continuo (CGA)}

Este método de optimización emplea un enfoque continuo del GA convencional propuesto por Chu \& Beasley en (Chu and Beasley 1997). Utiliza los operadores de selección, recombinación y mutación con una representación continua para generar la población que representa los tamaños de los GDs. El algoritmo genético tiene cuatro características principales: generación de la población inicial, implementación de operadores genéticos para generar la población descendiente, generación de la nueva de población y verificación de los criterios de parada. Todos ellos son importantes para resolver cualquier problema de optimización, y se explican a continuación.

\section{Población inicial:}

Este es el primer paso del proceso iterativo que compone el CGA, igual que al $\mathrm{BH}$, se propone una población con un tamaño de $n_{i}$ filas y $n_{g d}$ columnas, utilizando la ecuación (8) para obtener los individuos que componen la población inicial. Después de generar la población inicial, la etapa esclava evalúa la función adaptación de todos los individuos que forman $P_{t}$, seleccionando al individuo con el valor más bajo de la función adaptación como la mejor solución (Incumbente). 


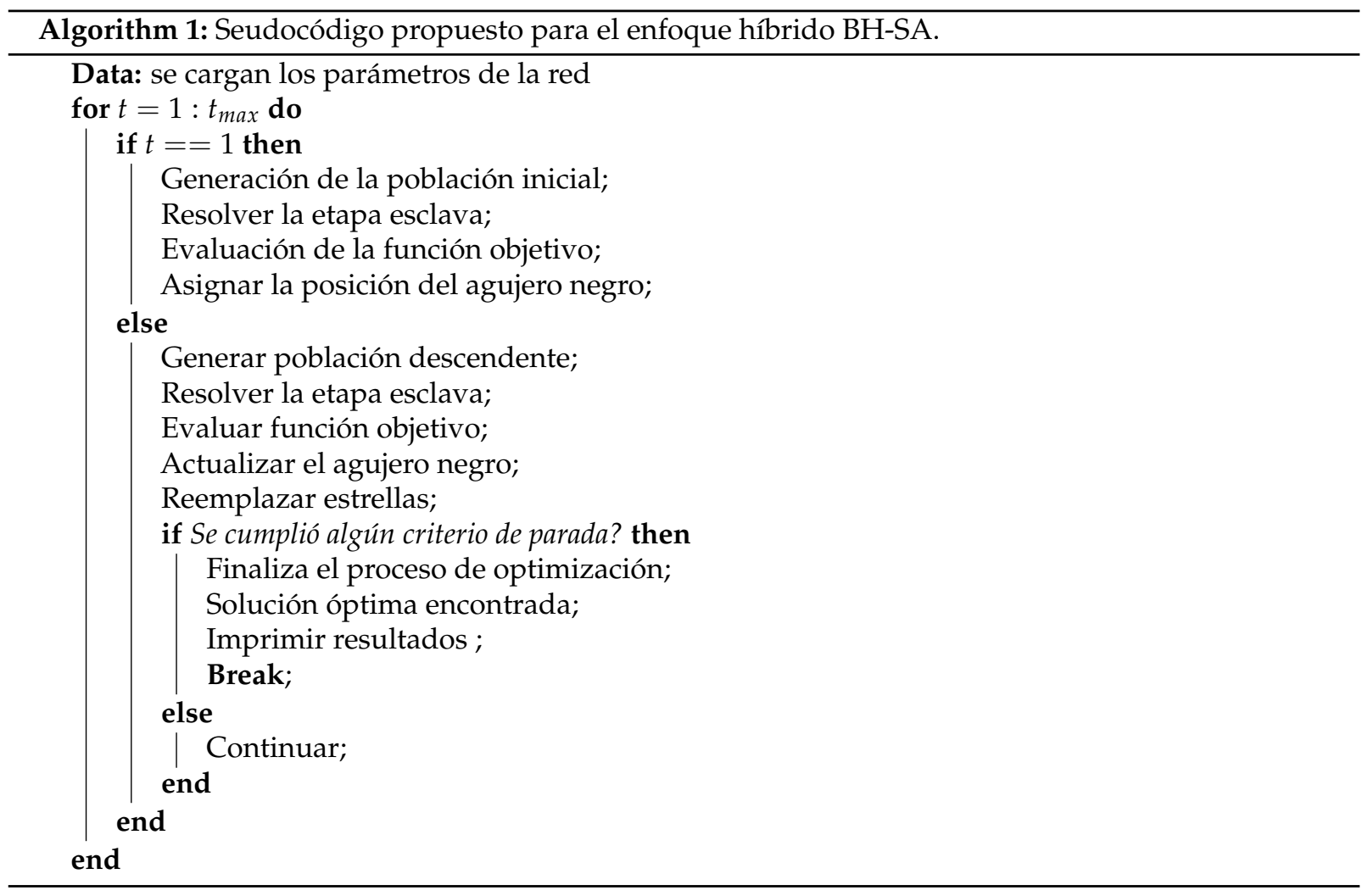

\section{Población descendiente:}

El algoritmo genético corresponde a un proceso de optimización iterativo, por lo tanto, es necesario generar nuevas soluciones potenciales para reemplazar las malas soluciones de la población actual. Para generar esos nuevos individuos, los operadores clásicos de selección, recombinación y mutación se adaptan para resolver la optimización continua.

\section{Selección:}

La población descendiente comienza a seleccionar un subconjunto arbitrario de individuos contenidos en la población actual, en esta selección se elige un número aleatorio $r$ entre 1 a $n_{i}$, es decir, $r=1+\left(n_{i}-1\right)$ rand, donde rand corresponde a un número aleatorio entre 0 y 1 . Si $r<n_{i}$, una matriz adicional $\left(n_{i}-r\right) \times n_{g d}$ con soluciones potenciales se genera mediante el uso de la misma estrategia adoptada para la población inicial. El conjunto total de individuos seleccionados está conformado por la combinación de ambas estrategias.

\section{Recombinación:}

Este proceso altera la población descendiente a través del siguiente principio. Si la probabilidad de recombinación $r_{p}$ es mayor que $50 \%$ (valor determinado heuristicamente), entonces, dos individuos arbitrarios (seleccionados aleatoriamente) se recombinan en una posición arbitraria seleccionada mediante un número aleatorio entre 1 a $n_{g d}$ PS. Si $r_{p}$ es inferior a $50 \%$, se promedian dos individuos arbitrarios (elegidos al azar) para generar un nuevo individuo potencial. Esta operación siempre genera 
individuos factibles, ya que la población inicial y las soluciones aleatorias se generan dentro de la región de admisibilidad de los generadores distribuidos. Este proceso continua hasta obtener la población descendente $\operatorname{con} n_{i}$ soluciones potenciales.

\section{Mutación:}

En este punto se explora la probabilidad de mutación $m_{p}$, es decir, si $m_{p}$ es mayor que $50 \%$ (valor que se determino heuristicamente), una posición arbitraria de la solución entre 1 y $n_{g d}$ se modifica mediante un valor de generación de energía arbitrario que garantiza que (4) se cumple. Si $m_{p}$ es inferior a 50\%, la solución potencial no se modifica. Este proceso continua hasta que se analizan todos los individuos descendientes. Una vez que la población descendiente ha sido generada, sus valores de función de aptitud se calculan utilizando la etapa esclava.

\section{Nueva población:}

En la nueva población se guardará el conjunto de las mejores soluciones encontradas por el algoritmo genético hasta la iteración actual $t$. Luego, se genera una nueva población combinando el conjunto actual y descendiente de individuos, lo que produce una población con $2 * n_{i}$ soluciones potenciales; si dos soluciones potenciales son idénticas, entonces, una de ellas se elimina. Este procedimiento se repite hasta garantizar que todas las posibles soluciones son diferentes (criterio de diversidad). Después de ese proceso, la lista de soluciones potenciales resultantes está ordenada de forma ascendente en términos de los valores de la función de aptitud, y las primeras soluciones potenciales $n_{i}$ se seleccionan como la nueva población para el próximo ciclo de iteración $t+1$. Finalmente, la incumbente es actualizada.

\section{Criterio de parada:}

El algoritmo genético continuo propuesto finaliza su proceso de optimización con las mismas condiciones propuestas para el $\mathrm{BH}$. Finalmente, el Algoritmo 2 presenta el proceso iterativo de la metodología híbrida CGA / SA.

\subsubsection{Optimización por enjambre de partículas (PSO)}

El PSO es un algoritmo metaheurístico bioinspirado basado en el comportamiento de las bandadas de peces y aves, y fue propuesto por Eberhart y Kennedy en 1995 (Kennedy and Eberhart 1995). Este método aprovecha la técnica utilizada por los grupos de animales para explorar una región y encontrar una fuente común de alimento para todos los individuos del grupo. Al modelar a cada individuo como una partícula, es posible transformar el grupo de individuos en un enjambre de partículas disperso sobre un espacio de solución. Este enjambre de partículas está limitado por un conjunto de restricciones asociadas con cada problema. En el algoritmo PSO, cada paso o iteración considera la información asociada a cada partícula, así como la información del enjambre de partículas, para generar el siguiente movimiento. El desarrollo computacional ejecutado por el PSO para resolver el problema de OPF en redes CC se resume en el Algoritmo ?? y se explica a continuación. 


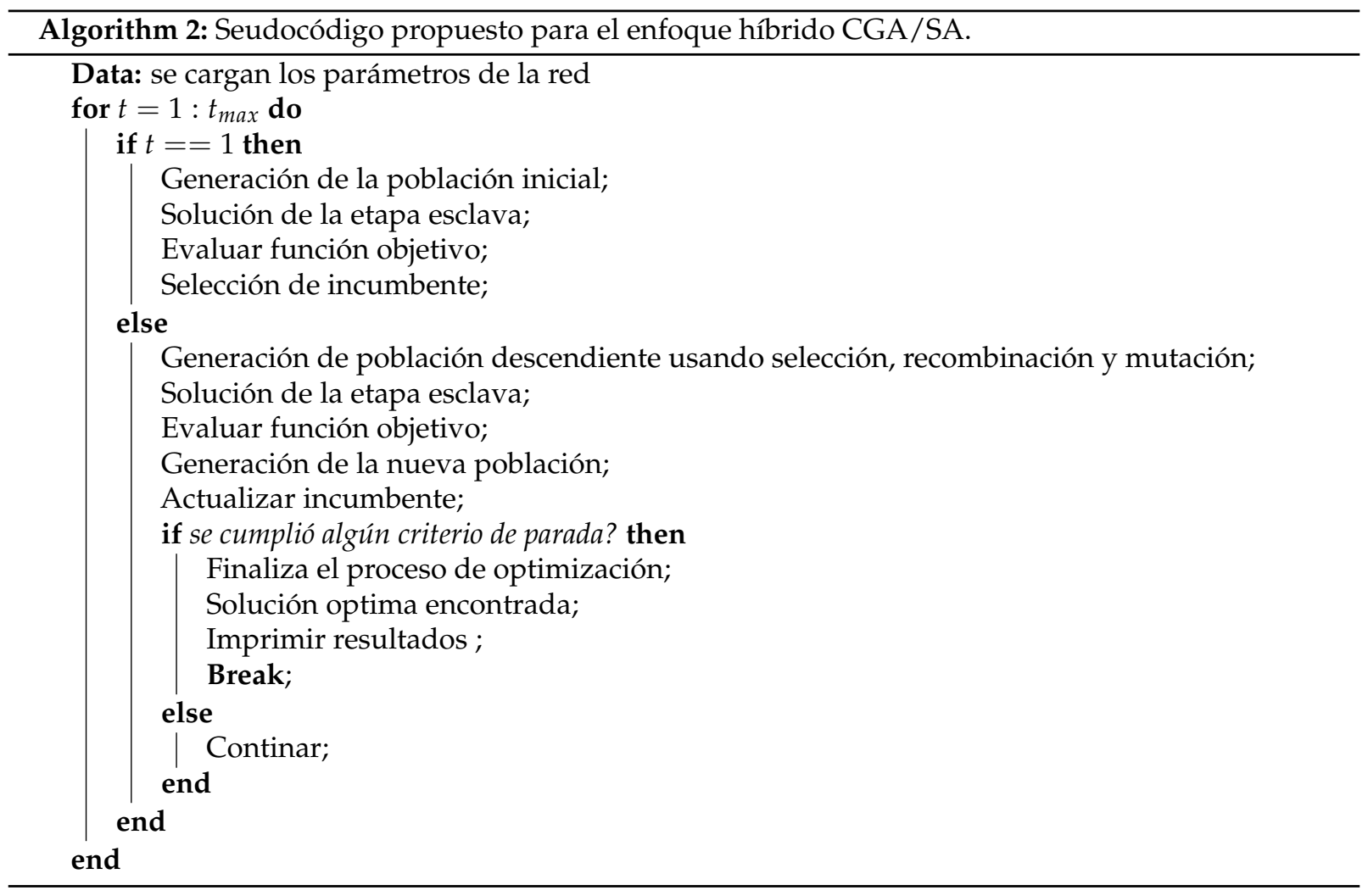

\section{Generación del enjambre de partículas:}

En la primera iteración, el enjambre de partículas $\left(P_{t}\right)$ se genera de manera aleatoria, asignando la potencia activa que deben suministrar los GDs como en el caso del BH y CGA (usando la ecuación (8)). En el PSO, cada individuo de la población se conoce como partícula y la población como enjambre de partículas. En este método de optimización, el número de partículas $(P)$ depende del problema particular (Wang et al. 2018). Posteriormente, se resuelve el flujo de potencia y se evalúa la función de aptitud para cada partícula utilizando la etapa esclava. Finalmente, se selecciona la mejor solución y mejor posición para cada partícula: bestpos ${ }_{i}$ y bestsol ${ }_{i}$, respectivamente; de igual manera, se detecta la mejor solución y la mejor posición del enjambre de partículas (Incumbente): bestpos $g$ y bestsol $l_{g}$.

\section{Movimiento de las partículas:}

El PSO usa un vector de velocidad para controlar el movimiento de las partículas en el espacio de solución. Para cada partícula, el movimiento en la iteración $t\left(x^{t}\right)$, se encuentra en función de la posición del enjambre de partículas en la última iteración $\left(x^{t-1}\right)$ y su velocidad de movimiento en la iteración actual $t\left(M S^{t}\right)$, como se muestra en la ecuación (12).

$$
x_{t}=x^{t-1}+M S^{t}
$$

En la primera iteración del proceso de optimización, la velocidad de movimiento para todas las partículas del enjambre (MS) se obtiene utilizando la ecuación (13); donde, $M S^{\min }$ y $M S^{\max }$ corresponden 
a los valores mínimos y máximos permitidos para la velocidad de movimiento de cada partícula, mientras que $o\left(P, n_{g d}\right)$ y $r\left(P, n_{g d}\right)$ representa una matriz rectangular llena de unos y números aleatorios entre cero y uno. A partir de la segunda iteración, se utiliza la ecuación (14) para calcular la velocidad de movimiento de cada partícula en la iteración $t\left(M S_{i}^{t}\right)$, la cual esta en función del factor de inercia en la iteración $t\left(\Omega^{t}\right)$, la velocidad de movimiento de la partícula en la iteración anterior $\left(M S_{i}^{t-1}\right)$, la posición de la partícula en la última iteración $\left(x_{i}^{t-1}\right)$, los factores cognitivo y social $\left(\phi_{1}\right.$ y $\left.\phi_{2}\right)$ que controlan la dirección del movimiento de la partícula considerando la mejor posición de cada partícula y el enjambre; y dos valores aleatorios $\left(r_{1}\right.$ y $r_{2}$ ) que evitan que la técnica quede atrapada óptimos locales (Wang et al. 2018).

$$
\begin{gathered}
M S=M S^{\min } o\left(P, n_{g d}\right)+\left(M S^{\max }-M S^{\min }\right) r\left(P, n_{g d}\right) \\
M S_{i}^{t}=\Omega^{t} M S_{i}^{t-1}+\phi_{1} r_{1}\left(\text { Bestpos }_{i}-x_{i}^{t-1}\right)+\phi_{2} r_{2}\left(\text { Bestpos }_{g}-x_{i}^{t-1}\right)
\end{gathered}
$$

Es importante resaltar que, el factor de inercia es el encargado de controlar la convergencia del PSO, este valor se actualiza en cada iteración utilizando el valor de iteración actual $(t)$, el número máximo de iteraciones propuestas para el algoritmo $\left(t_{\max }\right)$ y el límite max́imo y mínimo fijados a $\Omega$. La ecuación (15) permite calcular el valor del factor de inercia $\left(\Omega^{t}\right)$ en la iteración $t$.

$$
\Omega^{t}=\Omega^{\max }-\left(\left(\Omega^{\max }-\Omega^{\min }\right) / \Omega^{\max }\right) * t
$$

\section{Evaluación y actualización de la función adaptación}

Después del movimiento de las partículas, se evalúa la función de adaptación para cada partícula; y con esta información se actualiza la mejor solución y posición para cada partícula y del enjambre (la mejor solución del problema).

\section{Criterio de parada:}

Para el algoritmo PSO, se adoptan los mismos criterios de parada utilizados por el BH y CGA. El Algoritmo 3 representa el modo de operación del PSO para resolver el problema de flujo de potencia óptimo.

\subsection{Etapa esclava: aproximaciónes sucesivas (SA)}

El problema esclavo se utiliza para determinar la función adaptación y factibilidad de cada posible solución propuesta por la etapa maestra. En otras palabras, la etapa esclava calcula las variables eléctricas necesarias para estimar las pérdidas de potencia del sistema y verificar el cumplimiento de conjunto de restricciones que representan el problema. Para ese propósito, en este artículo se propone resolver el problema del flujo de energía a través del método iterativo basado en Aproximaciónes Sucesivas (SA), propuesto y analizado detalladamente en (Montoya et al. 2019; Montoya et al. 2018b), que permite resolver el problema de flujo de potencia en redes de CC ya sea de topología radial o mallada, proporcionando tiempos de procesamiento cortos. Estas características mejoran el rendimiento de las metodologías maestro-esclavo propuestas en documento. El método de SA emplea la siguiente ecuación para resolver el problema de PF: 


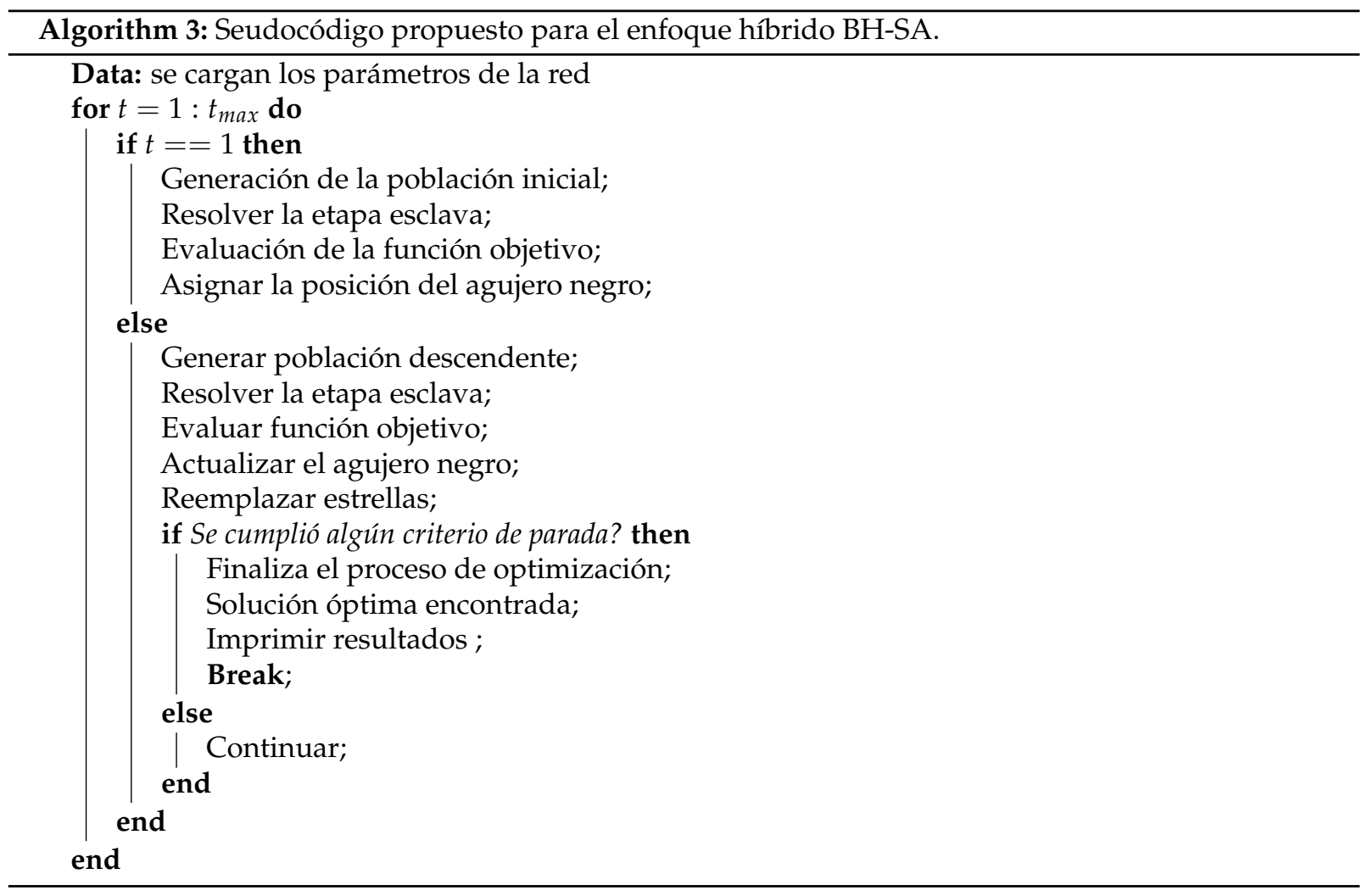

$$
G_{d d} v_{d}=-D_{d}^{-1}\left(v_{d}\right) P_{d}-G_{g d} v_{g}
$$

Donde $G_{d d}$ y $G_{g d}$ representan la matrix de conductancia asociadas a los nodos de potencia constante y generación, respectivamente. Las cuales son una matriz positiva simétrica definida, utilizada para obtener una expresión que permite resolver el problema no lineal aqui analizado. $V_{g}$ y $V_{d}$ representan los voltajes en los generadores y en los nodos de potencia constante, respectivamente. Finalmente, $P_{d}$ representa las potencias demandadas o inyectadas en los nodos de potencia constante. Para resolver el problema de PF, se simplifica la ecuación, obteniendo finalmente la siguiente expresión matemática:

$$
v_{d}=-G_{d d}^{-1}\left[D_{d}^{-1}\left(v_{d}\right) P_{d}+G_{g d} v_{g}\right]
$$

Reescribiendo la ecuación (17), de tal manera que se pueda resolver por medio de un proceso iterativo, la ecuación se modifica de la siguiente manera:

$$
v_{d}^{t+1}=-G_{d d}^{-1} D_{d}^{-1}\left(v_{d}^{t}\right) P_{d}-G_{d d}^{-1} G_{g d} v_{g},
$$

En este documento se resuelve la Ecuación (18) de manera iterativa hasta cumplir con un error de convergencia de $1 \times 10^{-10}$ (criterio de parada), empleando como punto de inicio un voltaje plano en todos los nodos del sistema (1 p.u).

\section{Resultados de la Simulación}

Para validar las metodologías de solución propuestas en este documento, se utilizaron dos sistema de prueba, de 21 y 69 nodos, descritos en (Garcés 2018) y (Grisales-Nore ña et al. 2018), respectivamente. 
Table 1. Parámetros de las técnicas de dimensionamiento.

\begin{tabular}{|c|c|c|c|}
\hline Método & CGA & $\overline{\mathrm{BH}}$ & PSO \\
\hline $\begin{array}{l}\text { Número de } \\
\text { partículas }\end{array}$ & 30 & 30 & 30 \\
\hline $\begin{array}{l}\text { Método de } \\
\text { selección }\end{array}$ & Torneo & $\begin{array}{c}\text { Radio de horizonte } \\
\text { de eventos }\end{array}$ & $\begin{array}{c}\text { Componente cognitivo } \\
\text { social: } 1.4\end{array}$ \\
\hline $\begin{array}{l}\text { Actualizar } \\
\text { método de } \\
\text { población }\end{array}$ & $\begin{array}{l}\text { Promedios: } \\
\text { cruzados }\end{array}$ & $\begin{array}{l}\text { Componente } \\
\text { social } \\
\text { y cognitivo }\end{array}$ & $\begin{array}{c}\text { Velocidad/Inercia } \\
\text { (max-min): } \\
(0.1-0.1) /(0.7-0.001)\end{array}$ \\
\hline Mutación & Población aleatoria & Población aleatoria & $\mathrm{R} 1$ = R2: aleatorio \\
\hline $\begin{array}{c}\text { Criterio de } \\
\text { parada }\end{array}$ & $\begin{array}{c}\text { Iteraciones Max. } \\
(200) \\
\text { Iteraciones de } \\
\text { no mejora: } \\
(50)\end{array}$ & $\begin{array}{c}\text { Iteraciones Max. } \\
(200) \\
\text { Iteraciones de } \\
\text { no mejora: } \\
(50)\end{array}$ & $\begin{array}{c}\text { Iteraciones Max. } \\
(200) \\
\text { Iteraciones de } \\
\text { no mejora: } \\
(50)\end{array}$ \\
\hline
\end{tabular}

Dado que el objetivo de estas metodologías es determinar el dimensionamiento óptimo de los generadores distribuidos (OPF), los sistemas de prueba se modificaron cambiando los nodos de generación con cargas de potencia constantes, pero manteniendo el mismo nivel de potencia asignado en el sistema de prueba tradicional para la generación. En este trabajo, la ubicación de los generadores distribuidos en la red eléctrica se basó en las ubicaciones informadas en la literatura para ambos sistemas de prueba, las especificaciones se presentan en las subsecciones 4.1 y 4.2. Los parámetros seleccionados para las técnicas de dimensionamiento se muestran en la Tabla 1. Los cuales fueron hallados de forma heurística con base en el análisis reportado en (Grisales-Nore ña et al. 2018), con el objetivo de proporcionar una comparación equitativa entre los métodos de solución. De esta manera, se consideró una población de 30 individuos, un máximo de 200 iteraciones y un contador de no mejora de 50 iteraciones como criterio de parada para todos los métodos.

Adicionalmente, se consideraron tres niveles diferentes de penetración de potencia máxima para cada GD en ambos sistemas de prueba: $20 \%, 40 \%$ y $60 \%$ de la potencia total demandada en el nodo Slack sin considerar GDs en la red eléctrica. Esto se hizo con el fin de evaluar el impacto de la potencia distribuida inyectada en la red CC. En este trabajo, cada GD instalado en la red eléctrica puede inyectar la potencia máxima permitida, siempre y cuando los demas generadores no estén inyectando energía en la red. Como parámetros para la etapa esclava, se asignó un número máximo de iteraciones igual a 2000, y un error de convergencia igual a $1 \times 10^{-10}$.

Finalmente, para calcular los tiempos de procesamiento promedio y la desviación mínima, media y estándar de la solución obtenida por los métodos de solución, cada escenario de prueba se ejecutó 1000 veces. Las simulaciones se llevaron a cabo en una estación de trabajo Dell Precision T7600 con 32 GB de memoria RAM y con una CPU Intel (R) Xeon (R) ES-2670 a 2.50 GHz.

\subsection{Sistema de prueba de 21 nodos}

En la versión modificada del sistema de 21 nodos, el nodo Slack genera una potencia equivalente a 5.8160 p.u., lo que representa una pérdida de potencia igual a 0.27603 p.u; ese valor corresponde al caso base (sin los GDs instalados en el sistema eléctrico). Las ubicaciones de los generadores en el sistema de prueba de 21 nodos se establecieron según lo reportado en (Mirjalili 2015), que corresponden a los nodos 9 , 12 y 16. 
Table 2. Resultados obtenidos en el sistema de 21 nodos

\begin{tabular}{|c|c|c|c|c|c|}
\hline \multicolumn{6}{|c|}{ Sistema de 21 nodos } \\
\hline \multirow{2}{*}{ Metodo } & \multirow{2}{*}{$\begin{array}{c}\text { Ubicación DG } \\
\text { /Dimesionamiento }\end{array}$} & \multicolumn{3}{|c|}{ Pérdidas de potencia } & \multirow{2}{*}{ Tiempo [s] } \\
\hline & & $\min [\mathbf{p . u}]$ & $\mu$ [p.u] & $\sigma[\%]$ & \\
\hline \multicolumn{6}{|c|}{ Generación max al 20\% = 1.1632 [p.u.] } \\
\hline \multirow{3}{*}{ BH } & $9 / 0.0063$ & & & & \\
\hline & $12 / 0.1932$ & 0.1321 & 0.1431 & 3.0163 & 0.2268 \\
\hline & $16 / 0.9616$ & & & & \\
\hline \multirow{3}{*}{ CGA } & $9 / 0.0069$ & & & & \\
\hline & $12 / 0.1752$ & 0.1320 & 0.1344 & 0.8459 & 0.3347 \\
\hline & $16 / 0.9805$ & & & & \\
\hline \multirow{3}{*}{ PSO } & $9 / 0$ & & & & \\
\hline & $12 / 0.1781$ & 0.1318 & 0.1330 & 4.0893 & 0.6644 \\
\hline & $16 / 0.9851$ & & & & \\
\hline \multicolumn{6}{|c|}{ Generación max al 40\% = 2.3264 [p.u.] } \\
\hline \multirow{3}{*}{ BH } & $9 / 0.2341$ & & & & \\
\hline & $12 / 0.7789$ & 0.0614 & 0.0675 & 4.9706 & 0.2711 \\
\hline & $16 / 1.3117$ & & & & \\
\hline \multirow{3}{*}{ CGA } & $9 / 0.3124$ & & & & \\
\hline & $12 / 0.7085$ & 0.0612 & 0.0622 & 0.9829 & 0.3707 \\
\hline & $16 / 1.3050$ & & & & \\
\hline \multirow{3}{*}{ PSO } & $9 / 0.3058$ & & & & \\
\hline & $12 / 0.7296$ & 0.0612 & 0.0623 & 5.2991 & 0.6663 \\
\hline & $16 / 1.2910$ & & & & \\
\hline \multicolumn{6}{|c|}{ Generación max al 60\% = 3.4896 [p.u.] } \\
\hline \multirow{3}{*}{ BH } & $9 / 0.8948$ & & & & \\
\hline & $12 / 1.0584$ & 0.0288 & 0.0319 & 7.2049 & 0.2390 \\
\hline & $16 / 1.4754$ & & & & \\
\hline \multirow{3}{*}{ CGA } & 9/1.0005 & & & & \\
\hline & $12 / 1.0667$ & 0.0280 & 0.0288 & 1.8535 & 0.3596 \\
\hline & $16 / 1.4219$ & & & & \\
\hline \multirow{3}{*}{ PSO } & $9 / 0.9330$ & & & & \\
\hline & $12 / 1.0739$ & 0.0279 & 0.0283 & 5.0709 & 0.6829 \\
\hline & $16 / 1.4827$ & & & & \\
\hline
\end{tabular}

La tabla 2 presenta los resultados obtenidos por las metodologías propuestas para resolver el problema de OPF en el sistema de prueba de 21 nodos. La información presentada en esta tabla está organizada de la siguiente manera: la primera columna presenta el método; la segunda columna muestra la ubicación de los GDs y la potencia inyectada por cada uno. Entre las columnas tercera a quinta se presentan el valor mínimo ( $\min [\mathrm{pu}])$, el valor medio $(\mu[\mathrm{pu}])$ y la desviación estándar $(\sigma[\%])$ de la función adaptación. Finalmente, la sexta columna presenta el tiempo promedio requerido por cada método. La figura 1 presenta la reducción mínima y media en las pérdidas de potencia obtenida por los métodos de solución. En la subfigura 1 (a) se presenta la reducción mínima de pérdidas de potencia obtenida por cada método para todos los niveles de generación permitidos a los GDs. En esta ilustración se puede apreciar que el método PSO proporciona la mejor solución, con una reducción promedio del $73.32 \%$ en $P_{\text {loss }}$, cuando se compara con el caso base. Además, se obtiene una mejora en la reducción mínima promedio en las $P_{\text {loss }}$ equivalente a $0.18 \%$ y $0.04 \%$ con respecto al BH y CGA, respectivamente. La subfigura 1 (b) muestra la reducción media en $P_{\text {loss }}$. En el caso particular cuando se considera una generación de GD máxima del 20\%, el PSO obtuvo los mejores resultados con una reducción media igual al 51,8\%; que es $3.65 \%$ y $0.5 \%$ más alto que BH y CGA. Para el caso de una generación máxima del $40 \%$, el CGA presenta la 

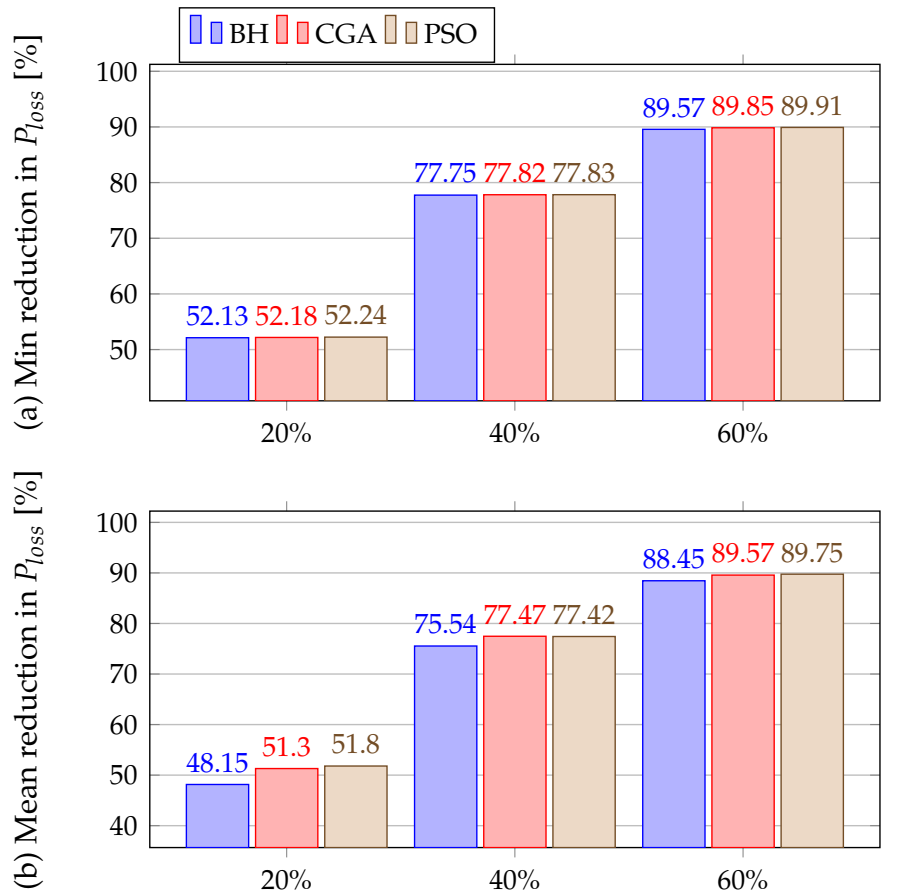

Figure 1. Reducción de pérdidas de potencia en el sistema de prueba de 21 nodos.

mejor solución con una reducción de 77.46 \%, obteniendo una mejora en la reducción media de 1.93\% y $0.05 \%$ con respecto a $\mathrm{BH}$ y PSO, respectivamente. Finalmente, para un valor de $60 \%$ en la generación de potencia por los GDs, la mejor solución se logra con el PSO (89.74\%), la segunda y tercera mejores soluciones son CGA $(89.57 \%)$ y BH $(88.45 \%)$. Analizando los resultados anteriores, el algoritmo PSO proporciona los mejores resultados promedio en términos de reducción mínima $(0.11 \%)$ y media $(1.24 \%)$ de pérdidas de potencia para el sistema de prueba de 21 nodos; cuando se compara con el CGA y BH, respectivamente. Además, la desviación estándar de las soluciones, dadas en la Tabla 2, muestran que todos los métodos exhiben una alta consistencia en los resultados, ya que $\sigma$ es inferior al $8 \%$ en todos los escenarios de prueba. También se puede apreciar que cuando la generación máxima permitida a los GDs aumenta, $\sigma$ disminuye para todos los métodos.

En la Figura 2 se analizan los tiempos de procesamiento requeridos por los métodos de solución, donde el $\mathrm{BH}$ presenta los tiempos de procesamiento más cortos en todos los escenarios con una reducción promedio de $30.8 \%$ y $63.39 \%$ con respecto al CGA y PSO. El CGA obtuvo la segunda mejor solución, presentando una reducción del 47,10\% en comparación con el PSO.

\section{2. sistema de prueba de 69 nodos}

Posterior a la adaptación realizada al sistema de 69 nodos, estableciendo solo cargas en sus nodos, se determinó que la potencia total generada en el nodo Slack es igual a 40.4311 p.u., y que el nivel de pérdidas de energía es igual a 1.5385 p.u, cuando no se consideran GDs instalados en la red de CC (caso base para este escenario). Todo lo anterior consiederando que el nodo Slack opera con un voltaje de 1 p.u. Posterior al análisis del caso base, en este sistema de prueba, los generadores son ubicados en los nodos 26, 61 y 66, como se informa en (Grisales-Nore ña et al. 2018).

La tabla 3 presenta los resultados de los métodos de solución aplicados al sistema de prueba de 69 nodos. Analizando los datos presentados en esta tabla, la Figura 3 muestra la reducción de pérdidas 


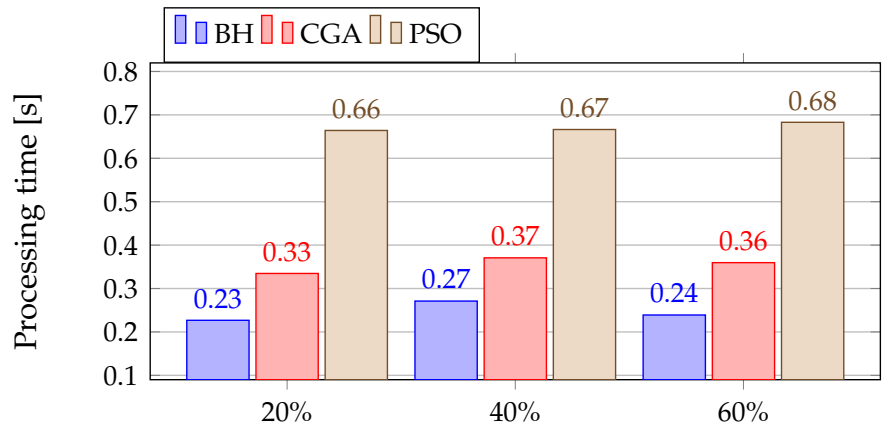

Figure 2. Rendimiento del tiempo de procesamiento para el sistema de prueba de 21 nodos
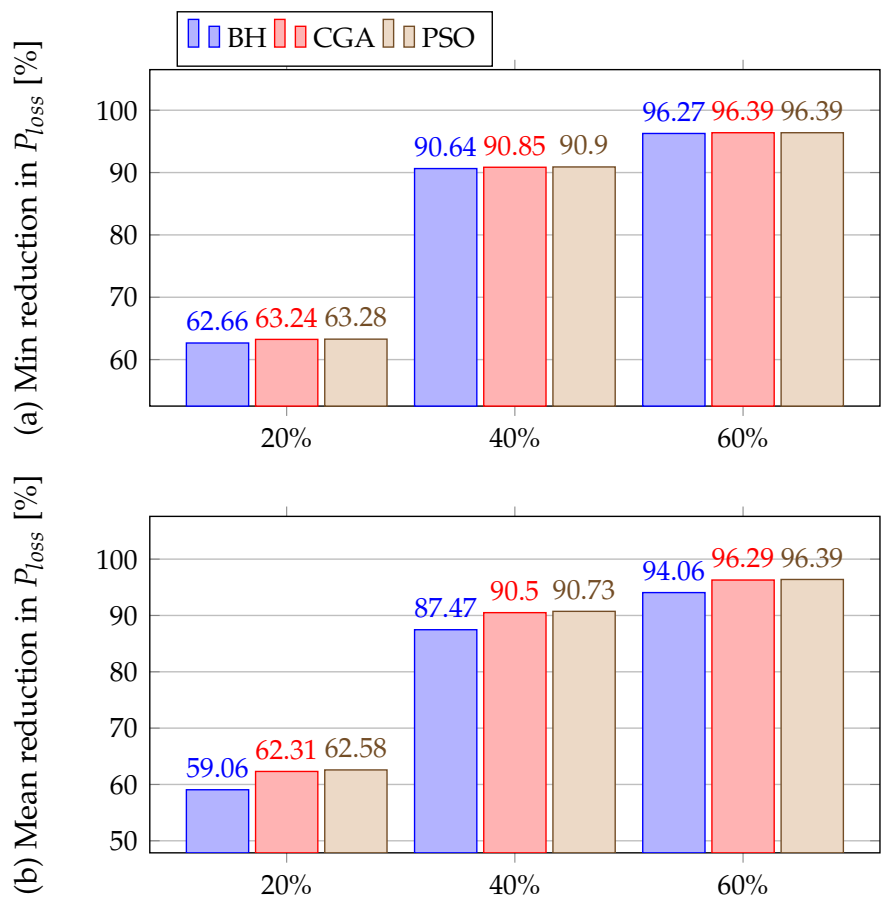

Figure 3. Reducción de pérdidas de potencia en el sistema de prueba de 69 nodos.

de potencia mínima y media, donde el PSO presenta los mejores resultados en todos los escenarios de prueba, presentando una mejora de $0.03 \%$ y $0.33 \%$ en la reducción de pérdidas de potencia mínima cuando se compara con el CGA y BH, respectivamente. Del mismo modo, para la reducción de la pérdidas de potencia media, el PSO presenta una reducción media del 0,02\% y 3,03\% mayor que CGA y BH, respectivamente. Con respecto al $\sigma$, similar al sistema de prueba de 21 nodos, el valor obtenido no excede el 7\%. Es importante resaltar que, a medida que aumenta el nivel máximo de generación de potencia, $\sigma$ también aumentan para BH y CGA; mientras que el $\sigma$ asociado al PSO disminuye. Los resultados anteriores confirman que el PSO proporciona la mejor solución para los sistemas de prueba de nodo 21 y 69, y el BH obtuvo los peores resultados en términos de calidad de la solución.

La figura 4 muestra los tiempos de procesamiento requeridos por los métodos de solución para el sistema de 69 nodos. En esta figura se puede observar que el BH está en primer lugar, y el CGA y el PSO están en el segundo y tercer lugar en términos de tiempo de procesamiento, respectivamente: el BH presenta una reducción promedio del tiempo de procesamiento de 45.72 \% y $69.11 \%$ con respecto al CGA y PSO. El tiempo de procesamiento requerido por los métodos para los sistemas de prueba 21 y 69 nodos 
Table 3. Results in the 69 bus test system

\begin{tabular}{|c|c|c|c|c|c|}
\hline \multicolumn{6}{|c|}{69 Node Test System } \\
\hline \multirow{2}{*}{ Metodo } & \multirow{2}{*}{$\begin{array}{c}\text { Ubicación DG } \\
\text { /Dimensionamiento }\end{array}$} & \multicolumn{3}{|c|}{ Pérdidas de potencia } & \multirow{2}{*}{ Ttiempo $[s]$} \\
\hline & & $\min$ [p.u] & $\mu$ [p.u] & $\sigma[\%]$ & \\
\hline & \multicolumn{5}{|c|}{ Generación max al 20\% = 8.0862 [p.u.] } \\
\hline \multirow{3}{*}{ BH } & $26 / 0.1618$ & & & & \\
\hline & $61 / 4.5862$ & 0.5745 & 0.6298 & 4.8000 & 0.9845 \\
\hline & $66 / 3.2877$ & & & & \\
\hline \multirow{3}{*}{ CGA } & $26 / 0.0040$ & & & & \\
\hline & $61 / 5.7967$ & 0.5655 & 0.5798 & 1.1088 & 1.8391 \\
\hline & $66 / 2.2798$ & & & & \\
\hline \multirow{3}{*}{ PSO } & $26 / 0.0002$ & & & & \\
\hline & $61 / 5.5722$ & 0.5649 & 0.5757 & 8.7501 & 3.6141 \\
\hline & $66 / 2.5139$ & & & & \\
\hline \multicolumn{6}{|c|}{ Generación max al 40\% = 16.1724 [p.u.] } \\
\hline \multirow{3}{*}{ BH } & $26 / 1.6018$ & & & & \\
\hline & $61 / 12.8098$ & 0.1440 & 0.1928 & 13.0986 & 0.9717 \\
\hline & $66 / 1.6554$ & & & & \\
\hline \multirow{3}{*}{ CGA } & $26 / 1.7335$ & & & & \\
\hline & $61 / 12.1362$ & 0.1407 & 0.1462 & 2.5630 & 1.8502 \\
\hline & $66 / 2.2840$ & & & & \\
\hline \multirow{4}{*}{ PSO } & $26 / 1.5487$ & & & & \\
\hline & $61 / 12.1981$ & 0.1399 & 0.1426 & 3.5541 & 3.5253 \\
\hline & $66 / 2.4256$ & & & & \\
\hline & \multicolumn{5}{|c|}{ Generación max al 60\% = 24.2586 [p.u.] } \\
\hline \multirow{3}{*}{ BH } & $26 / 4.0141$ & & & & \\
\hline & $61 / 15.3273$ & 0.0575 & 0.0913 & 24.9146 & 1.1902 \\
\hline & $66 / 2.3043$ & & & & \\
\hline \multirow{3}{*}{ CGA } & $26 / 3.7186$ & & & & \\
\hline & $61 / 16.0335$ & 0.0556 & 0.0571 & 2.1793 & 2.1079 \\
\hline & $66 / 2.3446$ & & & & \\
\hline \multirow{3}{*}{ PSO } & $26 / 3.7511$ & & & & \\
\hline & $61 / 15.8844$ & 0.0556 & 0.0556 & $7.3 \times 10^{-7}$ & 3.0462 \\
\hline & $66 / 2.4575$ & & & & \\
\hline
\end{tabular}

permite identificar que los métodos con el mejor rendimiento (método más rápido) y el peor rendimiento (método más lento) en términos de tiempos de procesamiento son el BH y PSO; lo cual es lo opuesto al resultado obtenido en términos de calidad en la solución.

La figura 5 presenta las diferentes relaciones obtenidas por cada metodología con respecto a las pérdidas de potencia y tiempo de procesamiento, para cualquier tamaño de red y el nivel máximo de generación de potencia por parte de los GDs considerado en este trabajo. En esta figura, el eje Y presenta el valor promedio de la pérdidas de potencia media, en porcentaje, con respecto a los casos base (sin GD); mientras que el eje $\mathrm{X}$ informa el tiempo de procesamiento promedio de cada método, también en porcentaje, con respecto al método híbrido que requiere el tiempo de procesamiento más largo en todos los sistemas de prueba, es decir, PSO. En ambos ejes, los valores promedio se calcularon considerando los resultados obtenidos en todos los sistemas de prueba. De la Fig. 5, se observa que el BH es el método más rápido, al requerir solo $36.60 \%$ del tiempo total utilizado por el PSO; pero con la peor respuesta en terminos de reduccion de pérdidas de potencia media (24,64\%). El CGA obtuvo el segundo lugar en términos de tiempo de procesamiento y pérdidas de potencia promedio, con un tiempo de procesamiento promedio de $23.52 \%$ y una pérdidas de potencia promedio de $22.09 \%$. Finalmente, la peor solución en 


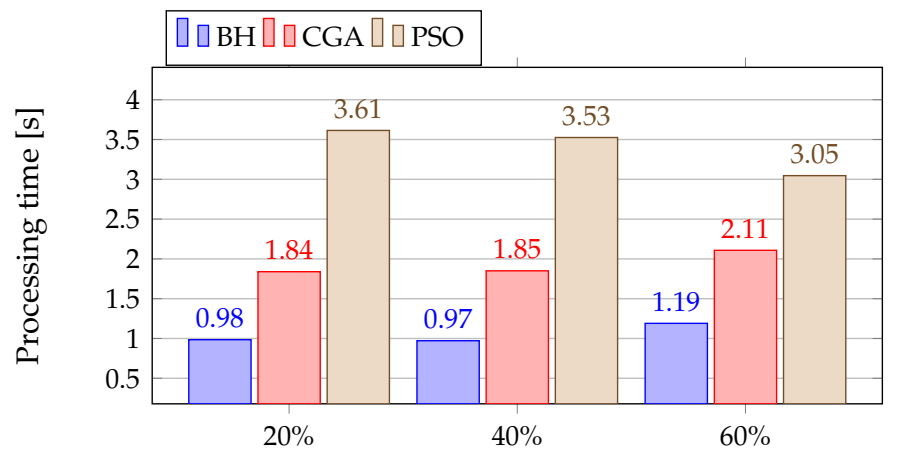

Figure 4. Tiempo de procesamiento requerido por el sistema de prueba 69 nodos

términos de tiempo de procesamiento es el método PSO, con un tiempo de procesamiento promedio de 2.03s, sin embargo, obtuvo la mejor solución en términos de pérdidas de potencia promedio, con un valor de $(21.88 \%)$. Con esos resultados se concluye que el mejor equilibrio entre la reducción de la pérdidas de potencia y el tiempo de procesamiento lo obtiene el BH, seguido del CGA y el PSO.

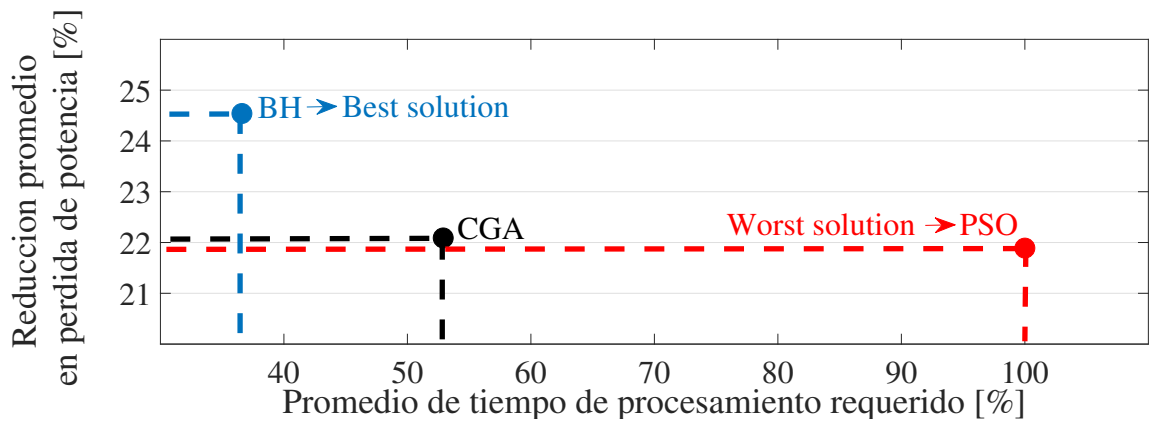

Figure 5. Impacto de las metodologías híbridas en todos los sistemas de prueba y niveles de generación de GD.

\section{Conclusiones}

En este trabajo se estudió el problema del flujo de potencia óptimo en redes de CC utilizando diferentes escenarios de penetración de potencia para los GDs: 20\%, 40\% y 60\% de la potencia total generada por el nodo Slack sin la presencia de GDs. Los sistemas de prueba empleados son versiones modificadas de los sistemas de prueba de 21 y 69 nodos. Esos escenarios de prueba fueron propuestos con el objetivo de evaluar el impacto de la generación de energía distribuida en el sistema eléctrico en términos de pérdidas de energía, los tiempos de procesamiento requerido y la robustez de cada método de solución propuesto en este artículo. En este sentido, se propuso una metodología maestro-esclavo que combina tres métodos de optimización continua: BH, CGA y PSO, con el método de SA empleado para resolver el problema de flujo de potencia en redes de CC; para resolver el problema de OPF en redes CC. La etapa maestra se encarga de definir la potencia óptima que cada GD debe inyectar para reducir la pérdidas de potencia. La etapa esclava es responsable de evaluar la función de adaptación de cada posible solución proporcionada por la etapa maestra, resolviendo el problema del flujo de potencia a través de la SA. La selección de la SA se basó en el excelente rendimiento proporcionado por este en términos de calidad de la solución y tiempos de procesamiento. 
Los resultados obtenidos por los métodos de solución en ambos sistemas de prueba muestran que el PSO ofrece la mejor solución en términos de reducción de pérdidas de potencia. Sin embargo, este método requiere tiempos de procesamiento más largos en comparación con el BH y el CGA. El BH presentó los tiempos de procesamiento más cortos en todos los casos bajo análisis, pero también la peor solución en términos de reducción de pérdidas de potencia. Se analizaron las relaciones proporcionadas por cada metodología en términos de pérdidas de potencia y tiempo de procesamiento. Concluyendo que la metodología $\mathrm{BH} / \mathrm{SA}$ propuesta en este trabajo presenta la mejor relación entre la reducción de pérdidas de potencia y tiempo de procesamiento. Por esa razón, entre los enfoques analizados en este estudio, la metodología híbrida $\mathrm{BH} / \mathrm{SA}$ se considera el método más adecuado para resolver el problema de flujo de potencia óptimo en redes de CC de cualquier tamaño y nivel de generación GD.

Es importante resaltar que la selección del software Matlab se realizó con el objetivo de evaluar la efectividad de las metodologías propuestas en un software reconocido en la literatura, y de esta forma fortalecer la calidad de los resultados obtenidos por las metodologías propuestas. Sin embargo, es importante mencionar que a pesar de que se cambie la herramienta de simulación, todos los resultados obtendrán porcentajes similares al comparar su efectividad en términos de la calidad de la solución y tiempo de procesamiento. Por lo cual los resultados de esta investigación son adecuados para la selección del metodo con la mejor eficiencia para resolver el problema abordado en este manuscrito. Como trabajo futuro, la ubicación óptima y el dimensionamiento de los recursos energéticos distribuidos, como la generación renovable y los sistemas de almacenamiento de energía, pueden abordarse mediante el uso de algoritmos híbridos similares. Además, las metodologías híbridas propuestas en este artículo se pueden usar en aplicaciones de control de microrredes para determinar el punto de ajuste de los controladores en función de las condiciones de operación de las cargas y los recursos de energía distribuida.

\section{Soporte financiero}

Esta investigación fue financiada por el Instituto Tecnológico Metropolitano, la Universidad Nacional de Colombia y Colciencias bajo el proyecto "Estrategia de transformación del sector energético Colombiano en el horizonte de 2030 Energética 2030","Generación distribuida de energía eléctrica en Colombia a partir de energía solar y eólica" (Código: 58838, Hermes: 38945).

Author Contributions: Conceptualización, L.F.G.-N and O.D.G.-R; Metodología, L.F.G.-N and C.A.R.-P.; Investigación, L.F.G.-N. and J.A.O.-T.; Escritura, revisión y Edición, M.A.R.-C., and O.D.G.-R.

\section{References}

Bouchekara, H. (2014). Optimal power flow using black-hole-based optimization approach. Applied Soft Computing, 24:879 - 888. doi:https://doi.org/10.1016/j.asoc.2014.08.056.

Bouchekara, H. R. E. H. (2013). Optimal design of electromagnetic devices using a black-hole-based optimization technique. IEEE Trans. Magn., 49(12):5709-5714. doi:10.1109/TMAG.2013.2277694.

Chu, P. and Beasley, J. (1997). A genetic algorithm for the generalised assignment problem. Computers E Operations Research, 24(1):17 - 23. doi:https:/ /doi.org/10.1016/S0305-0548(96)00032-9.

Garces, A. (2017). Uniqueness of the power flow solutions in low voltage direct current grids. Electric Power Systems Research, 151:149 - 153. doi:https:// doi.org/10.1016/j.epsr.2017.05.031.

Garcés, A. (2018). On the convergence of newton's method in power flow studies for dc microgrids. IEEE Transactions on Power Systems, 33(5):5770-5777. doi:10.1109/TPWRS.2018.2820430.

Gil-González, W., Montoya, O. D., Holguín, E., Garces, A., and ña, L. F. G.-N. (2019). Economic dispatch of energy storage systems in dc microgrids employing a semidefinite programming model. Journal of Energy Storage, 21:1 - 8. doi:https:/ /doi.org/10.1016/j.est.2018.10.025.

Grisales-Nore ña, L. F., Gonzalez Montoya, D., and Ramos-Paja, C. A. (2018). Optimal sizing and location of distributed generators based on pbil and pso techniques. Energies, 11(4). doi:10.3390/en11041018. 
Grisales-Noreña, L. F., Garzon-Rivera, O. D., Danilo Montoya, O., and Ramos-Paja, C. A. (2019). Hybrid metaheuristic optimization methods for optimal location and sizing dgs in dc networks. In Figueroa-García, J. C., Duarte-González, M., Jaramillo-Isaza, S., Orjuela-Cañon, A. D., and Díaz-Gutierrez, Y., editors, Applied Computer Sciences in Engineering, pages 214-225, Cham. Springer International Publishing.

Grisales-Noreña, L. F., Garzon-Rivera, O. D., Ramírez-Vanegas, C. A., Montoya, O. D., and Ramos-Paja, C. A. (2020). Application of the backward/forward sweep method for solving the power flow problem in DC networks with radial structure. Journal of Physics: Conference Series, 1448:012012. doi:10.1088/1742-6596/1448/1/012012.

Hasan, Z. and El-Hawary, M. E. (2014). Optimal Power Flow by Black Hole Optimization Algorithm. In 2014 IEEE Electrical Power and Energy Conference, pages 134-141. doi:10.1109/EPEC.2014.43.

Kennedy, J. and Eberhart, R. (1995). Particle swarm optimization. In Proceedings of ICNN'95 - International Conference on Neural Networks, volume 4, pages 1942-1948 vol.4. doi:10.1109/ICNN.1995.488968.

Li, J., Liu, F., Wang, Z., Low, S. H., and Mei, S. (2018). Optimal power flow in stand-alone dc microgrids. IEEE Transactions on Power Systems, 33(5):5496-5506. doi:10.1109/TPWRS.2018.2801280.

Mirjalili, S. (2015). The ant lion optimizer. Advances in Engineering Software, 83:80 - 98. doi:https:/ /doi.org/10.1016/j.advengsoft.2015.01.010.

Montoya, O. D., Garrido, V. M., Gil-González, W., and Grisales-Noreña, L. F. (2019). Power flow analysis in dc grids: Two alternative numerical methods. IEEE Transactions on Circuits and Systems II: Express Briefs, 66(11):1865-1869. doi:10.1109/TCSII.2019.2891640.

Montoya, O. D., Gil-González, W., and Garces, A. (2019). Sequential quadratic programming models for solving the OPF problem in DC grids. Electr. Power Syst. Res., 169:18-23. doi:10.1016/j.epsr.2018.12.008.

Montoya, O. D., Gil-González, W., and Grisales-Noreña, L. F. (2018a). Optimal Power Dispatch of DGs in DC Power Grids: a Hybrid Gauss- Seidel Genetic-Algorithm Methodology for Solving the OPF Problem. WSEAS Transactions on Power Systems, 13:335 - 346.

Montoya, O. D., Grisales-Noreña, L., González-Montoya, D., Ramos-Paja, C., and Garces, A. (2018b). Linear power flow formulation for low-voltage de power grids. Electric Power Systems Research, 163:375 - 381. doi:https:/ /doi.org/10.1016/j.epsr.2018.07.003.

Moradi, M. and Abedini, M. (2012). A combination of genetic algorithm and particle swarm optimization for optimal dg location and sizing in distribution systems. International Journal of Electrical Power and Energy Systems, 34(1):66 - 74. doi:https:/ / doi.org/10.1016/j.ijepes.2011.08.023.

Nasir, M., Iqbal, S., and Khan, H. A. (2018). Optimal planning and design of low-voltage low-power solar dc microgrids. IEEE Transactions on Power Systems, 33(3):2919-2928. doi:10.1109/TPWRS.2017.2757150.

Piotrowski, A. P., Napiorkowski, J. J., and Rowinski, P. M. (2014). How novel is the novel black hole optimization approach? Information Sciences, 267:191 - 200. doi:https:/ /doi.org/10.1016/j.ins.2014.01.026.

Velasquez, O., Giraldo, O. M., Arevalo, V. G., and Grisales-Noreña, L. F. (2019). Optimal power flow in direct-current power grids via black hole optimization. Advances in Electrical and Electronic Engineering, 17(1).

Wang, P., Zhang, L., and Xu, D. (2018). Optimal Sizing of Distributed Generations in DC Microgrids with Lifespan Estimated Model of Batteries. In 2018 21st International Conference on Electrical Machines and Systems (ICEMS), pages 2045-2049. doi:10.23919/ICEMS.2018.8549448.

(C) 2020 by the authors. Licensee TESEA, Cartagena, Colombia. This article is an open access article distributed under the terms and conditions of the Creative Commons Attribution (CC BY) license (http:/ / creativecommons.org/licenses/by/4.0/). 